\title{
sur la gestion et les finances publiques En Octobre 2020
}

\section{BUDGET DE L'ÉTAT ET DES OPÉRATEURS}

\section{$\pi$ Politique budgétaire}

Le soutien aux entreprises touchées par la crise

En précisant les conditions de mise en œuvre du couvre-feu instauré à partir du 16 octobre pour 4 semaines dans 8 métropoles, le Premier Ministre et le Ministre de l'Économie et des Finances ont annoncé le 15 octobre une série d'aides supplémentaires pour les secteurs les plus touchés par cette mesure : restauration, spectacle, événementiel... L'aggravation de la situation sanitaire ayant conduit à l'annonce par le Président de la République, le 29 octobre d'un « reconfinement national » jusqu'au $1^{\text {e décembre, }}$ de nouvelles mesures ont dû être présentées le lendemain. Ce nouveau volet, qui représente une enveloppe totale de $15 \mathrm{Md}$ est plus particulièrement ciblé sur les TPE, notamment les indépendants du commerce et de l'artisanat, qui vont être très touchés par la limitation de la circulation et de l'activité. Les aides du Fonds de solidarité pour les entreprises de moins de 50 salariés et les indépendants frappés de fermeture vont être portées à $10000 €$ par mois à partir du ler novembre, l'aide de $1500 €$ étant maintenue pour les entreprises restées ouvertes mais ayant perdu plus de $50 \%$ de leur chiffre d'affaires. Les conditions de l'activité partielle ( $84 \%$ du salaire net pour le salarié et remboursement intégral pour l'entreprise) seront maintenues jusqu'au $1^{\text {er }}$ janvier 2021 pour toutes les entreprises ayant dû fermer, même s'il n'y a pas eu accord d'entreprise (condition nécessaire pour le régime de l'activité partielle de longue durée qui est le plus favorable). Une exonération totale de cotisations sociales pour toutes les entreprises de moins de 50 salariés fermées administrativement a été décidée. Le différé de remboursement pour les prêts garantis par l'État sera allongé d'un an (jusqu'en mars 2022) et la possibilité de prêts d'État pour des entreprises n'ayant trouvé aucune solution sera ouverte à hauteur de $10000 €$ pour des entreprises de moins de 10 salariés. Enfin un crédit d'impôt de 30 \% va être mis en place pour les bailleurs qui accepteront d'annuler une partie de leurs loyers.

Tout savoir sur les relations financières avec I'UE

L'annexe au PLF 2021 sur les relations financières avec l'Union européenne fait apparaître que la contribution de la France va augmenter en 2021 de $25 \%$, de 21,5Md€ en 2020 à 26,9Md€. D'après les chiffres de 2019, la France est le deuxième contributeur derrière l'Allemagne. Sa contribution représente $10,5 \%$ de ses ressources fiscales nettes nationales et 15,7\% du budget de l'Union. Elle est le deuxième bénéficiaire en volume derrière la Pologne mais le 23e si on tient compte du nombre d'habitants. Avec 6,8Md€ d'excédent de versement par rapport aux dépenses de l'UE réalisées sur le territoire, elle est le troisième contributeur net derrière l'Allemagne (-14,3 Md€ et la Grande-Bretagne -6,8 Md€). L'annexe contient une mine de renseignements sur les finances de I'Union (par exemple, le cadre financier pluriannuel 2021-2027, I'accord du 21 juillet 2020 et le plan de relance Next Generation EU, le budget vert européen, le volet budgétaire du Brexit, les ressources propres, les compensations et rabais, les réponses à la crise sanitaire, la gestion et le contrôle des fonds structurels, la procédure de décharge, les enjeux et les programmes du budget par rubriques, le mécanisme des restes à liquider...).

\section{$\lambda$ Dette publique}

Dette : le cri d'alarme de Pierre Moscovici

Dans une interview accordée au journal «Aujourd'hui » le 3 novembre 2020, le Premier 
président de la Cour des comptes a estimé $q^{\prime} u^{\prime}$ " entre le coût de la crise sanitaire et les effets de la crise économique (la dette publique) a augmenté de l'ordre de 6500 euros par Français ». De plus, « la dette a autant augmenté en quelques mois qu'au cours des dix années précédentes. Il est nécessaire de garder notre crédit, relancer la croissance, maîtriser à moyen terme nos finances publiques. Si on ne le fait pas, la dette publique peut exploser à $140 \%$ du PIB ».

\section{$\pi$ Patrimoine de l'État}

\section{Le jaune relatif à l'État actionnaire}

Le rapport « jaune » relatif à l'État actionnaire, publié le 12 octobre, rappelle que 85 entités se situent dans le giron de l'Agence des participations de l'État. Le montant des opérations de cessions ou assimilées réalisées entre juin 2019 et juin 2020 équivaut à 3, $054 \mathrm{Md} €$. Parmi les faits marquants de l'année 2019, il y a l'introduction réussie de la Française des jeux (FDJ) sur le marché d'Euronext Paris. Le rapport rappelle que «l'État conserve un contrôle étroit de la FDJ, avec un pouvoir d'agrément des dirigeants et de tout actionnaire prenant + de $10 \%$ du capital ». L'autre fait marquant a été la création du grand pôle financier autour de la Caisse des dépôts. Dans son panorama général, le document revient également sur l'impact de la crise sanitaire du Covid sur certaines entreprises stratégiques et l'accompagnement de ces entreprises durant et après la crise.

Le résultat des enchères des fréquences $5 \mathrm{G}$

Comme l'a indiqué le site de l'Autorité de régulation des communications électroniques, des postes et de la distribution de la presse (ARCEP), suite à la dernière phase d'attribution d'enchères, les quatre opérateurs nationaux se sont engagés à payer un total de 2,786 Md € pour les $310 \mathrm{MHz}$ à attribuer. Au final, les enchères françaises ont donc remporté moins que les enchères réalisées en Allemagne et en Italie (6,55 Md€).

Le rapport d'activité de l'Agence des participations de l'État

Selon le rapport d'activité d'APE 2019-2020, publié le 14 octobre, la valorisation du portefeuille de participation dans des entreprises cotées a chuté de 30,3\% sur un an. La valorisation boursière du portefeuille coté de l'État s'établirait désormais à 52,3 Md€ en juin 2020. La valeur totale du portefeuille de l'APE est estimée à 84,5 Md€ au 30 juin 2020. En comparaison, la valeur du portefeuille était estimée à 112,7 Md€ au 31 décembre 2019, la valorisation boursière du portefeuille côté de l'État s'établissant à 74, 2 Md€.

\section{त Budget des opérateurs}

\section{La Cour des comptes veille sur Notre- Dame}

La Cour des comptes a réalisé un premier bilan de l'utilisation des fonds issus de la gestion des dons (825 M€) occasionnés suite à l'incendie de Notre-Dame de Paris. Dans son rapport, la Cour a estimé que cette gestion n'était pas suffisamment transparente. Cette gestion des dons déroge notamment au financement d'un établissement public (chargé de la conservation et de la restauration de la cathédrale). La juridiction financière estime que « faire reposer l'ensemble du financement de l'établissement sur les dons reversés au budget de l'État par voie de fonds de concours à partir desquels des subventions lui sont apportées apparaît comme une opération de complète débudgétisation très discutable dans son principe (...) mais plus essentiellement encore par rapport à la lettre et à l'esprit de la loi du 29 juillet 2019 ». La cour formule cinq recommandations pour améliorer la transparence de cette gestion. Elle propose notamment qu'au sein de l'établissement public soit mise en place une comptabilité analytique permettant une information détaillée sur l'emploi des fonds lié à la souscription nationale.

\section{$\lambda$ Administrations budgétaires}

\section{Le rapport d'activité la direction du budget}

Le rapport de la direction du budget (DB) sur l'année budgétaire 2019 a été présenté le 8 octobre. II dresse un panorama des finances publiques avant la crise sanitaire et économique et détaille le rôle de la direction du budget sur la mise en place des politiques publiques et de la réforme de l'État. La directrice du budget rappelle que « la responsabilité budgétaire » est au cœur du travail de la DB. À ce titre, le rapport rappelle que la responsabilité des gestionnaires est un des chantiers majeurs pour les années 2019, 2020 et suivantes. Le rapport revient aussi sur le centenaire de la direction du budget (v. Franck Mordacq, «Le centenaire de la direction du budget », Gestion \& Finances publiques, nov.-déc. 2019, p. 67).

\section{FinANCES LOCALES}

\section{Données générales}

\section{Le Sénat redéfinit l'autonomie financière dans la Constitution}

La proposition de loi constitutionnelle pour le plein exercice des libertés locales, adoptée par le Sénat le 20 octobre, aborde la question financière 
et suggère des compléments à l'article 72. Les sénateurs redéfinissent les ressources propres des collectivités, en excluant les recettes fiscales sur lesquelles elles n'exercent aucun pouvoir de taux ou d'assiette. En outre, le terme « déterminante » est remplacé par celui de « significative». La garantie financière en cas de transfert de compétence est calculée par le montant " estimé » de l'augmentation des dépenses et non plus par rapport aux dépenses passées de l'État, garantie qui doit faire l'objet d'un examen périodique défini par une loi organique.

\section{$\pi$ Fiscalité locale}

La compensation de la suppression de la TH

À l'heure où la fiscalité locale va à nouveau être débattue au Parlement, I'Institut des politiques publiques présente un rapport $n^{\circ} 27$ intitulé Suppression de la taxe d'habitation et réallocation de la fiscalité locale. Ce rapport réalisé à la demande de I'Assemblée des Communautés de France (AdCF) et l'Institut pour la recherche de la Caisse des Dépôts réexamine les conséquences de la nouvelle fiscalité mise en place à partir de 2023. La LFI 2020 prévoit de compenser les communes de cette perte en leur transférant le taux de TFPB des départements. En cas de surcompensation ou de sous-compensation, la loi prévoit un mécanisme de correction (les communes surcompensées devant verser leur excédant aux communes sous-compensées). Les EPCl et départements seraient quant à eux compensés par une fraction de TVA. Il apparaît que 69 \% des communes seraient surcompensées, d'où un effet assez intensif de I'utilisation du fameux coefficient correcteur. Le niveau de compensation des communes apparaît plus important dès lors que le territoire communal appartient à un département peu peuplé, à faible densité, et dont les résidents se caractérisent par des revenus relativement faibles et un taux de chômage élevé. Le rapport examine deux autres possibilités pour le niveau intercommunal : une fraction du taux de la TFPB départementale serait allouée aux $\mathrm{EPCl}$, soit de manière uniforme sur tout le territoire, soit de telle sorte que chaque EPCI reçoive une TFPB du département égale à son montant de TH perdu.

\section{Secteur public local}

Les finances des centres communaux et intercommunaux d'action sociale

Le nouveau «Cap sur... » n 13, octobre 2020, de l'Observatoire des finances et de la gestion publique locales s'attaque à une question méconnue : les enjeux financiers portés par les centres communaux et intercommunaux d'action sociale.
La loi NOTRe du 7 août 2015, qui rend leur création facultative dans les communes de moins de 1500 habitants a abouti à une diminution de leur nombre passant de plus de 28000 à moins de 15000 entités. Les dépenses totales consolidées (c'est-à-dire budget principal et budgets annexes) représentent environ 6,3 Md€ en 2019, dont 2,8 Md€ en 2019 pour les dépenses de fonctionnement avec la décomposition suivante : frais de personnel (63\%), achats et charges externes (23\%). Ce rapport relève toutefois l'existence de disparités importantes en matière de dépenses de fonctionnement s'expliquant par plusieurs facteurs : différences de moyens, choix politiques locaux en matière d'action sociale et périmètre d'intervention des CCAS, besoins des populations, rôle joué par les tiers (associations, autres niveaux de collectivités...).

\section{La territorialisation du plan de relance}

Le Premier ministre et le ministre de l'économie ont organisé un dispositif de territorialisation du plan de relance (v.infra management public)

\section{FinANCES SOCIALES}

\section{Deuxième conférence du dialogue social à Matignon}

Le Premier Ministre a réuni le 26 octobre l'ensemble des partenaires sociaux pour la deuxième conférence du dialogue social. La première conférence du 17 juillet avait ouvert 17 chantiers (v. Repères de juillet 2020), mais le calendrier de ceux-ci se trouve remis en cause par la deuxième vague de la pandémie. Sur plusieurs sujets, la concertation a été reportée : partage de la valeur dans l'entreprise, suivi de la réforme de la formation professionnelle, conditions de travail des salariés de la « deuxième ligne »...Seules les discussions sur le télétravail ont progressé. Le sort de la réforme des retraites n'a pratiquement pas été abordé, bien que restant officiellement à l'ordre du jour. La seule annonce significative a concerné la réforme de l'Assurance chômage, pour laquelle la mise en place du nouveau mode d'indemnisation a été une fois de plus repoussée de 3 mois, jusqu'au $1^{\circ}$ avril 2021.

\section{Politiques d'allègement des charges et coût du travail}

\section{Mesures de soutien aux entreprises}

Un nouveau train de mesures de soutien aux entreprises pénalisées par les décisions de couvrefeu et de reconfinement a été annoncé comprenant des assouplissement en matière de finance- 
ment des activités partielles et d'exonération de cotisations (v.supra Budget de l'État).

\section{$\lambda$ Dépense de santé / Hôpital / Assurance maladie}

\section{La Cour des Comptes critique la politique de santé}

La Cour des Comptes a publié le 5 octobre son rapport sur l'application des lois de financement de la Sécurité sociale dans le contexte inédit de creusement abyssal des déficits du fait de la crise du COVID-19. Cette situation rendra indispensable le transfert à la CADES de 136Md€ de dette née et à venir et la prolongation de l'existence de la Caisse jusqu'en 2033. Mais selon la Cour, le montant agrégé des déficits 2020 à 2024 excéderait d'au moins $50 \mathrm{Md} €$ le montant (92 Md€) repris à ce titre dans la dette CADES, du fait d'un taux de progression de I'ONDAM arrêté pour 2021 (+6\% hors COVID) et des mesures du "Ségur de la Santé ». La Cour recommande donc un infléchissement de la courbe de progression des dépenses de santé. En particulier, une attention prioritaire devrait être apportée à l'évolution des dépenses de médecine de ville pour lesquelles il n'existe pas de mécanisme de régulation infra-annuel. Les dépenses de prestations familiales se sont stabilisées, suite à la sous-indexation des revalorisations par rapport à l'inflation et à la modulation de leurs montants en fonction des revenus. Les retraites ont connu de multiples réformes depuis 1993, le principal enseignement en étant que le recul de l'âge légal a, à court terme, un effet plus important que l'allongement de la durée de cotisation. La Cour fait une douzaine de recommandations visant à améliorer l'efficience du système de santé, parmi lesquelles une révision des périmètres des groupements hospitaliers de territoires, un renforcement de la cohérence des financements spécifiques (MIGAC et FIR) qui viennent en complément de la tarification à l'activité, une régulation plus active des dépenses et des prescriptions des dispositifs médicaux. Elle propose également de mieux cibler les dépenses d'action sociale des CAF (crèches, centres de loisirs...) qui ont un caractère très redistributif. La Cour fait ensuite un certain nombre de propositions pour faire progresser la qualité de la gestion : réduire les erreurs ( $5 \mathrm{Md} \mathrm{f}$ de prestations), dont $90 \%$ correspondent à des trop perçus; à cette fin, intensifier les contrôles de cohérence de données, harmoniser les réseaux des caisses et favoriser les mutualisations. Enfin, la Cour fait un développement sur les URSSAF, dont le périmètre de recouvrement se sera accru de $110 \mathrm{Md} €$ entre 2018 et 2023, en particulier avec le transfert des cotisations AGIRC-ARRCO, qui est une opération particulièrement délicate.

Rapport de la Cour sur les groupements hospitaliers de territoires

À la demande de la Commission des Affaires sociales du Sénat, la Cour des Comptes a produit un rapport sur les Groupements hospitaliers de territoires, publié le 5 octobre, dont elle regrette qu'ils n'aient pas modifié fondamentalement les relations entre les établissements et que leur impact sur l'offre de soins n'ait pas été très significative.

\section{Nouvelles mesures en faveur de l'hôpital}

Le Ministre des Solidarité et de la santé a annoncé le 21 octobre de nouvelles mesures en faveur des personnels soignants : majoration de $50 \%$ des heures supplémentaires, indemnisation des congés non pris d'ici la fin de l'année, avancée dès décembre 2020 de la deuxième tranche de revalorisation du Ségur de la santé. Avec un effort supplémentaire de 2,4Md€ pour 2020 qui inclut les équipements et matériels destinés à faire face à la " $2^{\mathrm{e}}$ vague ", l'ONDAM hospitalier 2020 devrait atteindre $+9 \%$ en 2020 .

\section{$\pi$ Retraites}

\section{Déficits : projections très alarmistes du} COR

Dans une note d'étape transmise à la demande du Premier ministre, le Conseil d'orientation des retraites dresse le 15 octobre un tableau très pessimiste de l'évolution du système de retraite du fait de la crise du COVID-19 : chute massive des recettes de cotisation $(-5,2 \%$ en 2020$)$ suite à l'effondrement de la masse salariale et maintien d'uns croissance modérée des dépenses de pension. Le déficit des régimes serait de -25,4 Md€ en 2020 et de -13,3 Md€ en 2024 (dont 8,5 Md€ pour le seul régime général), soit 0,5 points de PIB, alors que le rapport du COR de l'an dernier prévoyait un déficit de -10 Md€.

\section{त Assurance chômage}

\section{Vers un déficit historique de I'UNEDIC}

Comme il avait été annoncé dans les Repères de septembre 2020, le bureau de I'UNEDIC a confirmé le 21 octobre un déficit du régime d'Assurance chômage de -18,7 Md€ en 2020, et une prévision de -9,7 Md€ en 2021, ce qui porterait la dette de I'UNEDIC à 65, 2 Md€ fin 2021. En 2020, ce déficit s'explique à hauteur de $57 \%$ par le financement de l'activité partielle incombant au régime, de $28 \%$ par l'augmentation du volume 
des allocations chômage et d'environ 15 \% par les reports de cotisation.

\section{$\pi$ Solidarité / Lutte contre la pauvreté / Revenu universel}

Un rapport sur la lutte contre la pauvreté au temps du COVID

France Stratégie a publié le 1er octobre la note d'étape du comité d'évaluation de la stratégie nationale de prévention et de lutte contre la pauvreté, qui fait le bilan de la situation après la première phase de la pandémie. Le comité souligne plusieurs aspects qui touchent plus particulièrement la population pauvre : conditions de logement, décrochage scolaire et universitaire, perte de contact avec les services sociaux, isolement des SDF... Tout en soulignant le rôle de filet de sécurité qu'a joué le système de protection sociale et l'ampleur des mesures prises par le gouvernement, la note pointe l'insuffisance des crédits fléchés sur le soutien aux personnes précaires ( $800 \mathrm{M} €$ dont $533 \mathrm{M} €$ pour la hausse exceptionnelle de l'allocation de rentrée scolaire), et le fait qu'il est nécessaire de recentrer la stratégie sur la situation des enfants, sur les conditions d'accès aux droits rendues plus difficiles par le confinement et sur la nécessité de s'attacher davantage aux personnes déjà touchées par la pauvreté, qui ont été moins bien soutenues que celles qui risquaient d'y entrer du fait de la perte de leurs revenus. Ces recommandations ont été en partie prises en compte dans les annonces faites par le gouvernement le 24 octobre (cf infra).

Les nouvelles mesures de solidarité annoncées par le gouvernement

En déplacement à Marseille, le Premier Ministre a annoncé le 24 octobre une nouvelle série de mesures pour lutter contre la pauvreté. Plus orientés sur l'accompagnement du retour à l'emploi que sur la revalorisation des minima sociaux, ces mesures-qui représentent une enveloppe de près de 2 Md€ jusqu'en 2022, ont été critiquées par les associations qui ont regretté en particulier l'absence de revalorisation du RSA. Celle-ci, comme I'avait annoncé le Président de la République le 14 octobre va se concentrer une aide de solidarité unique (comme celle versée en mai dernier) de $150 €$ aux bénéficiaires du RSA et de l'ASS (plus $100 €$ par enfant aux personnes au RSA, à I'ASS ou aux APL); elle est complétée par une aide de $150 €$ aux jeunes de moins de 25 ans non étudiants touchant l'APL et aux étudiants boursiers; à ce volet $(1,265 \mathrm{Md} €)$ s'ajoutent $100 \mathrm{M} €$ pour les associations et diverses actions destinées à faciliter l'accès effectif aux droits. Le déve- loppement des aides à l'activité représente une seconde enveloppe de 0,420Md€ principalement affectée au secteur de "l'insertion par l'activité économique » et aux " parcours emploi-compétence ». Enfin 0,213 Md€ seront dédiés à l'hébergement et au maintien dans le logement.

\section{L'aide et l'action sociale en France}

La DREES a publié le 30 les données 2018 de I'aide et de l'action sociale qui représentent une dépense annuelle de 38,4 Md€, dont la responsabilité relève pour moitié des départements. Cette aide est en légère baisse $(-0,2 \%)$ par rapport à 2017

\section{FISCALITÉ ET PROCÉDURE FISCALE}

Focus de la Cour des comptes sur la mission fiscale des Douanes

Un rapport thématique de la Cour des comptes porte sur les exercices 2013 à 2019 de la DGDDI, et notamment sur sa mission fiscale. Dans son rapport 2018, la Cour des comptes avait recommandé des efforts de modernisation et de simplification de la gestion fiscale de la DGDDI afin de mieux gérer la fraude aux prélèvements obligatoires. Cette réforme a bien été engagée - télédéclarations et télépaiement des taxes applicables aux alcools et tabacs et de l'octroi de mer, abrogation de 11 taxes obsolètes (taxes sur les appareils automatiques, sur les manifestations sportives, sur les farines, sur les céréales, sur la chaptalisation," les huiles alimentaires, sur l'édition des ouvrages de librairie) - mais la Cour des comptes estime que les missions fiscales de la DGDDI, gagneront à être transférées vers la DGFIP, non seulement en fusionnant les réseaux de recouvrement mais aussi en établissant des liaisons informatiques et enfin en révisant l'organisation du réseau fiscal et comptable. D'ores et déjà, le recouvrement de la fiscalité sur les alcools a été transféré par la loi de finances pour 2020 à la DGFIP à l'horizon 2024.

\section{Impôt sur le capital}

$2 e$ rapport du Comité d'évaluation des réformes de la fiscalité

Après une étude publiée le mois dernier sur le sujet (v. Repères de sept. 2020), et un premier rapport rendu l'an dernier (v. Repères de oct. 2019)، le comité d'évaluation des réformes de la fiscalité (France Stratégie), a remis le 8 octobre 2020 son $2^{e}$ rapport afin d'évaluer les dispositifs issus de la LFI 2018 (IFI et PFU de 30 \% sur les revenus 
du capital) supposés encourager les investissements dans les entreprises. D'une part, sur I'IFI, le Comité observe une baisse du nombre d'expatriations (163 départs en 2018 contre 700/900 sous les quinquennats précédents) et une hausse du nombre d'impatriations fiscales (250 retours en 2019). Pour la première fois depuis 2000, le nombre de retours de redevables excède celui du nombre de départs. Le coût budgétaire de cette réforme, 2,5 Md€ au lieu des 3,2 Md€ initialement annoncés (produit ISF, 4,1 Md€ en 2017), repose sur des recettes IFI supérieures aux prévisions (1,2 Md€ en 2018 et 1,6 Md€ en 2019 au lieu de $850 \mathrm{M} €$ attendus). Avec l'effet sur I'exil fiscal, c'est un gain budgétaire annuel de long terme, sans doute très supérieur, non évalué à ce jour (emplois, revenus, etc.). D'autre part, sur le PFU, le Comité constate des niveaux proches de la moyenne de I'UE pour les taux d'imposition sur les actifs mobiliers, ce qui a conduit à des hausses sensibles de dividendes versés en 2018 et 2019, qui ont généré d'importantes recettes fiscales. Quant au coût budgétaire de cette réforme (initialement estimé entre 0,8 à 0,9 Md€, hausse de CSG concomitante comprise), le Comité note qu'en définitive la perte d'IR pour l'État est nulle eu égard à cette hausse des dividendes. Pour mémoire, en 2013, la suppression du PFL et la mise au barème des dividendes, qui auraient dû engendrer un gain de recettes pour les finances publiques de $425 \mathrm{M€}$, avaient entraîné une contraction de la base fiscale (réduction de $70 \%$ des dividendes) et un coût budgétaire entre 1,1 et 2,1 Md€. Enfin, ce rapport souligne que le gain financier est conséquent pour les $0,1 \%$ des foyers fiscaux les plus aisés (38 000 foyers).

\section{$\lambda$ Impôt sur les sociétés et impôts de production}

L'adaptation de la fiscalité des entreprises à une économie mondialisée

Le rapport annuel du CPO pour 2020, dont le mandat de ses membres a été prolongé de trois mois du fait des circonstances (art. 45, L. n²020935, 30 juillet 2020 de finances rectificative pour 2020), a étudié la fiscalité directe des entreprises en France (IS et impôts de production). Lors de sa présentation devant les députés de la commission des finances, le Président du CPO Pierre Moscovici a estimé la marge de manœuvre fiscale étroite. II faut selon lui assurer la capacité de l'État à taxer les profits des entreprises pour que le financement du modèle social et la lutte contre les inégalités ne reposent pas que sur les ménages, sans compromettre la compétitivité et l'attractivité économique de la France. Selon le rapport, la France continue d'afficher un niveau et un nombre de prélèvements sur les sociétés plus élevés qu'au sein de la moyenne de l'OCDE, du fait de l'existence des impôts de production, spécificité française. Selon le CPO, cet ensemble hétéroclite de taxes fiscales assises sur les salaires, la valeur ajoutée, le chiffre d'affaires ou le foncier affecte la compétitivité des entreprises, tout en limitant l'assiette de I'IS. Le CPO estime que la baisse des impôts de production dans le PLF 2021 est une voie à emprunter, mais avec prudence, car il ne parait pas illogique non plus d'éviter une dégradation des finances publiques. Il faut selon lui indiquer et chiffrer les mesures structurelles de compensation, préalable indispensable à la poursuite de leur baisse. Concernant I'IS, il préconise de mener jusqu'à son terme la baisse du taux nominal d'IS d'ici 2022 tout en surveillant l'optimisation fiscale des entreprises et sa répartition entre les États, ce qui est difficile à déterminer. D'où, selon le $\mathrm{CPO}$, la nécessité d'achever le chantier de la réforme de la fiscalité mondiale des entreprises sous l'égide de l'OCDE. Les simulations réalisées par le CPO démontrent que l'impact du « pilier $n^{\circ} 1$ » de l'accord OCDE (répartition des droits d'imposition entre États de production et États de consommation) serait faiblement négatif pour la France $(0,4 \mathrm{Md})$ et que l'impact du « pilier $n^{\circ} 2$ » de l'accord de l'OCDE (un taux minimum d'imposition des résultats des entreprises multinationales de $12,5 \%$ ) pourrait générer des recettes fiscales supplémentaires (7 Md€). En cas d'échec des négociations, le CPO recommande de conclure des accords similaires au niveau de l'UE et de créer à cet échelon une taxe sur les services numériques sur le modèle mis en œuvre par la France (L. n 2019-759 du 24 juillet 2019 portant création d'une taxe sur les services numériques et modification de la trajectoire de baisse de I'IS).

\section{Dégrèvement exceptionnel de CFE «Covid-19 » en 2020}

Pour mémoire, le législateur a permis aux communes et $\mathrm{EPCl}$ à fiscalité propre d'octroyer une aide fiscale exceptionnelle, au titre de 2020, en faveur des entreprises de taille petite ou moyenne des secteurs du tourisme, de l'hôtellerie, de la restauration, de la culture, du transport aérien, du sport et de l'évènementiel qui ont été particulièrement affectés par le ralentissement de l'activité lié à l'épidémie de covid-19 (art. 11 L. n²020-935, 30 juillet 2020 de finances rectificative pour 2020). Sont ainsi éligibles les entreprises de ces secteurs réalisant moins de $150 \mathrm{M€}$ de chiffre d'affaires. Les collectivités territoriales peuvent ainsi instaurer, par une délibération 
adoptée entre le 10 juin et le 31 juillet 2020, une réduction de contribution foncière des entreprises à hauteur des $2 / 3$ de la cotisation établie au titre de 2020. Compte tenu du caractère exceptionnel de ce dispositif, celui-ci prendra la forme d'un dégrèvement dont le coût sera partagé à parts égales entre les collectivités territoriales et l'État. Le dispositif proposé s'applique uniquement aux cotisations dues au titre de 2020 et ne concerne pas les taxes additionnelles ni annexes à la CFE. La DGCL vient de publier la liste des communes et des EPCl ayant pris cette délibération, pour le rôle général de la taxation de CFE.

\section{Holding mixte et pacte Dutreil : la Cour de Cassation rejoint le Conseil d'État}

La Cour de Cassation vient de rendre un arrêt important concernant l'éligibilité à l'exonération du pacte Dutreil en matière transmission aux parts de sociétés holding ayant une activité mixte (Art. 787 B du CGI). La Cour de Cassation rejoint le Conseil d'État (CE, plén. 13 juin 2018, Req. $n^{\circ} 395495 ; C E, 8$ e et $3 e$ ch. réunies, 23 janv. 2020, Req. $n^{\circ} 435562$ ). Elle précise que l'exonération du pacte Dutreil s'applique aux sociétés dont l'activité professionnelle est principale. La prépondérance de l'activité professionnelle et ou civile s'apprécie en considération d'un faisceau $d$ 'indices déterminés d'après la nature de l'activité et les conditions de son exercice. Les titres d'une holding mixte sont éligibles à l'exonération du pacte Dutreil pour autant que l'activité d'animation est principale. Le caractère principal de l'activité d'animation de groupe doit être retenu « notamment lorsque la valeur vénale, au jour du fait générateur de l'imposition, des titres de ces filiales détenus par la société holding représente plus de la moitié de son actif total».

\section{Suppression des petites taxes fiscales à faible rendement budgétaire}

Un décret $n^{\circ} 2020-1207$ du 30 septembre 2020 relatif à la suppression de taxes à faible rendement à compter de 2020 (JORF n 0240 du 2 oct. 2020) vient tirer les conséquences, en matière réglementaire, de la suppression de certaines impositions prévues par le législateur en LFI 2019 (art. 26-2-III L. n²018-1317, 28 déc. 2018 de finances pour 2019 - taxe sur la publicité télévisée, taxe sur la publicité diffusée par voie de radiodiffusion sonore et de télévision, taxe sur la publicité diffusée par les chaînes de télévision) et en LFI 2020 (art. 21 et 22 L. n²019-1479, 28 déc. 2019 de finances pour 2020 - taxe sur les plus-values de cession de logements sociaux, taxe sur les activités commerciales non salariées à durée saisonnière, contribution perçue au profit de l'Institut national de prévention et d'éducation pour la santé, taxe sur les déclarations et notifications des produits du vapotage, taxe sur les premières ventes de dispositifs médicaux, droit fixe dû par les entreprises ferroviaires au profit de l'Autorité de régulation des transports, taxe sur les voyageurs de commerce, taxe annuelle sur la détention de véhicules polluants, droit de sécurité dû par les entreprises ferroviaire).

\section{$\pi$ Politique fiscale}

Suppression de la taxe d'habitation et réallocation de la fiscalité locale

(v. Finances locales).

\section{$\lambda$ Impôts sur le revenu}

La Conseil d'État confirme sa jurisprudence sur la domiciliation fiscale

Le fait de détenir des biens immobiliers dans un État ne peut seul fonder la domiciliation fiscale d'un contribuable dans cet État. En l'espèce, le Conseil d'État souligne que pour juger que les requérants avaient "le centre de leurs intérêts économiques » et, par suite, leur domicile fiscal en France, la CAA de Versailles s'est fondée sur la circonstance qu'ils possédaient dans cet État des sociétés et des biens immobiliers, et qu'elle a donc commis une erreur de droit (CE, $9^{e}-10^{e}$ ch. réunies, 07 oct. 2020, M. et Mme A., Req. $n^{\circ}$ 426124).

Le Conseil d'État annule une doctrine publiée au BOFIP au sujet du régime fiscal des impatriés

Le Conseil d'État vient de rendre un arrêt par lequel il annule le $\S 80$ et le $\S 90$ du BOFIP-Impôts (BOI-RSA-GEO-40-10-30-10), publié le 12 septembre 2012, excluant du champ d'application de l'exonération de l'article 155 B-II du CGI les impatriés qui ne disposent pas effectivement de revenus professionnels pour lesquels ils bénéficient de l'exonération d'IR prévue au I de cet article. Les requérants ont demandé l'annulation pour excès de pouvoir des commentaires publiés au BOFIP-Impôt. Le Conseil d'État vient de juger que ces paragraphes méconnaissaient les dispositions législatives qu'ils ont pour objet d'interpréter (CE, $8^{e}$ et $3^{e}$ ch. réunies, 21 oct. 2020, M. et et Mme B., Req. $n^{\circ}$ 442799). 


\section{Management PUBlic}

\section{$\lambda$ Politique de modernisation}

Les lauréats du Fonds de transformation de l'action publique

Le fonds pour la transformation de l'action publique est doté de $700 \mathrm{M} €$ sur 5 ans et a déjà mobilisé $580 \mathrm{M} €$ sur 97 projets. Le comité de sélection du fonds de transformation de l'action publique a arrêté le 6 octobre une liste de 19 nouveaux projets co-financés à hauteur de $112 \mathrm{M} €$. Parmi les projets retenus : le portail commun de recouvrement fiscal et social, la plate-forme d'assistance et d'orientation du guichet unique formalités, le Design System de l'État, Sciences comportementales et optimisation de l'action publique.

\section{Le projet de loi ASAP adopté}

L'Assemblée nationale a voté le 28 octobre, le projet de loi sur l'accélération et la simplification administratives (ASAP), tel qu'arrêté en commission mixte paritaire. Le projet, dont la procédure législative a été accélérée par le Gouvernement et retardée par la crise sanitaire, est passé de 50 à 150 articles (v. Repères de février 2020). Ce texte "fourre-tout » a été déféré au Conseil constitutionnel.

\section{$\lambda$ Administration numérique}

La feuille de route de Bercy sur les données

Le secrétariat général des ministères économiques et financiers a publié une "feuille de route » destinée à faire des données un outil de transformation de l'action publique. Elle comprend 25 actions réparties en trois axes : le capital humain, l'architecture des données, l'usage des données. Elles seront mises en œuvre sous l'impulsion du réseau des administrateurs directionnels de données

La Cour des comptes et les grands projets numériques de l'État

Dans un rapport destiné à la commission des finances du Sénat, rendu public le 15 octobre, la Cour des comptes constate que la conduite des grands projets numériques ne s'est pas beaucoup améliorée depuis les échecs de Louvois ou de I'ONP. Elle recommande une extension des compétences de la Direction du numérique (Dirnum), une plus grande mutualisation et un effort important en matière de ressources humaines.

\section{Rapport de l'AlFE}

Le rapport d'activité 2019 de l'Agence pour l'information financière de l'État rend compte de I'avancement de ses projets: généralisation de la facturation électronique, dématérialisation de la commande publique, amélioration du système d'information Chorus et extension du télétravail dans la fonction publique.

\section{Après Stop covid, Tousanticovid}

Le gouvernement a lancé le 22 octobre sa nouvelle application de traçage "Tousanticovid » qui prend le relais de Stop Covid-19 qui n'a pas eu le succès escompté (v. Repères Covid d'avril, mai, juin, juillet, octobre) : 3 millions de chargements en 5 mois pour 800 cas contacts signalés. Le nouveau dispositif est une simple amélioration du précédent avec plus d'informations (accès à la plateforme de conseils Mesconseilscovid, géolocalisation des centres de dépistage, accès direct aux formulaires d'autorisation de déplacement, statistiques) et devrait bénéficier d'un meilleur soutien des pouvoirs publics, notamment en communication. Une étude montrerait qu'un cas déclaré dans l'appli permet d'éviter à $5 \%$ des contacts de retransmettre le virus, ce niveau pouvant monter à $37 \%$ si un tiers de la population I'utilise. La nouvelle appli présente les mêmes avantages que la précédente (sécurité garantie) et les mêmes inconvénients (absence de compatibilité avec les autres appli des pays voisins). Elle a été téléchargée plus de 4 millions de fois en 10 jours.

\section{Dématérialisation, des objectifs ambitieux}

La ministre chargée de la transformation de l'action publique a présentée la nouvelle édition de l'observatoire de la dématérialisation qui assure le suivi des 250 démarches les plus courantes: $72 \%$ (182) sont réalisées pour un objectif de $100 \%$ en 2022. En revanche, $12 \%$ seulement des procédures sont accessibles aux personnes atteintes de handicap (objectif $80 \%$ ). La ministre a précisé la répartition des $500 \mathrm{M} €$ du plan de relance : $204 \mathrm{M} €$ pour l'innovation,208 M€ pour les outils numériques des agents, $88 \mathrm{M} €$ pour les projets des collectivités locales.

\section{$\lambda$ Administration territoriale}

Mise en oeuvre territorialisée du plan de relance

Le discours du Ministre de l'économie prononcé le 21 octobre 2020 détaille le plan de relance et I'implication des collectivités. Une circulaire du Premier ministre du 23 octobre en précise l'organisation. Elle repose sur les préfets de région 
et sur un comité régional de pilotage et de suivi coprésidé avec le directeur régional des finances publiques et, le cas échéant, le Président du Conseil régional. Les ministères devront déconcentrer une part importante des crédits. Des accords régionaux de relance, articulés avec les contrats de plan État-région, et des contrats infra régionaux seront conclus avec les collectivités territoriales. Certaines mesures ne peuvent être déclinées territorialement (baisse des impôts de production, allocation de rentrée scolaire ou la prime à l'embauche des jeunes). En revanche, dans de nombreux autres cas, sera mise en $œ u v r e$ une logique d'appels à projets, comme par exemple pour les 2 milliards d'euros de dotations de soutien à l'investissement local. Les montants inscrits dans ces accords ou contrats de relance resteront conditionnés à une mise en oeuvre « rapide et effective des mesures». En cas de retard, " des redéploiements pourront être effectués au niveau national ». Une enveloppe de 600 millions d'euros est déjà programmée dans le PLF pour 2021 en cours de discussion au profit des régions.

\section{$\pi$ Fonction publique}

Le jaune sur la fonction publique et les rémunérations

Le rapport annexe au projet de loi de finances relatif à la fonction publique et aux rémunérations contient toutes les statistiques utiles sur les 5,6 millions de fonctionnaires ( $+0,7 \%$ en un an).

La protection sociale complémentaire des fonctionnaires critiquée

Un rapport des trois inspections générales interministérielles dresse un bilan critique de la réforme de la protection sociale complémentaire des agents de la fonction publique qui repose sur le référencement $d$ 'organismes après mise en concurrence : faible intégration du sujet dans la conduite des ressources humaines, baisse du niveau de participation des ministères, très grandes hétérogénéité de ces contributions, fragilisation des mutuelles historiques, illisibilité du système... Les rapporteurs recommandent des adaptations du dispositif pour améliorer la couverture complémentaire des agents et augmenter la solidarité envers les retraités et les familles.

\section{Reconfinement : télétravail et/ou présence}

Une circulaire du 29 octobre de la ministre de la fonction et de la transformation publiques prescrit le développement du télétravail lorsqu'il est possible et la continuité de la présence sauf exceptions limitées ( contact à risque, personnes vulnérables, enfant de mois de 16 ans en l'absence de crèche ou d'école) ainsi que des mesures de protection des agents et des usagers.

\section{EUROPE}

\section{$\pi$ Budget européen}

Tribune des groupes du Parlement européen en faveur de la conditionnalité

Dans une tribune publiée le 6 octobre 2020, les présidents des quatre principaux groupes du Parlement européne, Dacian Ciolos, pour Renew Europe, Iratxe Garcia Perez pour Socialistes et democrates (S\&D), Ska Keller et Philippe Lamberts, pour les Verts/Alliance libre européenne et Manfred Weber pour le Parti populaire européen (PPE) rappellent que les valeur européennes «ne sont pas à vendre » et réclament une conditionnalité des versements européens au respect de l'État de droit, exigé par l'article 2 du Traité sur I'Union européenne. C'est la position adoptée par une résolution largement adoptée le mercredi 7 octobre, par 521 voix pour, 152 contre et 21 abstentions.

Blocage des négociations du cadre financier pluriannuel :

Le 8 octobre 2020, le Parlement européen a annoncé avoir interrompu la négociation avec les États membres sur le prochain budget pluriannuel de I'UE pour la période 2021-2027, en raison du refus des États membres d'augmenter les crédits européens. Après sept séances de négociations, la présidence allemande a proposé une augmentation de 9 milliards d'euros (sur un total de 1074 milliards d'euros) alors que le Parlement réclame 39 milliards d'euros de plus sur la période pour renforcer 15 programmes communautaires phare comme Erasmus, la recherche, la défense ou encore la santé ». Ce blocage institutionnel a pour effet de retarder la procédure d'approbation du plan de relance.

\section{Euro}

Le Président de la Bundesbank appelle à ne pas pérenniser le PSPP

Dans un entretien publiée le 7 octobre 2020, et traduit ici, Jens Weidmann, président de la Bundesbank, a déclaré que "Karlsruhe ne nous a pas délivré de chèque en blanc » en validant le programme d'achat de titres du secteur public (PSPP, Public sector purchase program). Selon lui « Si on assouplissait [ces mesures], il pourrait en découler un problème juridique. La flexibilité du PEPP est étroitement liée au fait qu'il a un objectif clairement limité et qu'il est restreint à la phase de 
crise. C'est pourquoi le PEPP [Pandemic Emergency Purchase Programme] ne doit pas devenir un dispositif permanent. »

\section{$\pi$ Fiscalité}

Mise à jour de la liste des pays et territoires non coopératifs à des fins fiscales

Le 6 octobre 2020, Le Conseil a décidé d'ajouter Anguilla et la Barbade à la liste de l'UE des pays et territoires non coopératifs à des fins fiscales. Les îles Caïmans et Oman ont été retirés de la liste après avoir adopté les réformes nécessaires. Désormais douze pays et territoires restent inscrits : les Samoa américaines, Anguilla, la Barbade, les Fidji, Guam, les Palaos, le Panama, le Samoa, les Seychelles, Trinité-et-Tobago, les Îles Vierges américaines et le Vanuatu.

\section{INTERNATIONAL}

\section{$\lambda$ Relations multilatérales}

Airbus-Boeing : I'OMC autorise I'UE à surtaxer les importations américaines

Le 15 octobre 2020, par une communication aux autorités européennes, confirmée par une réunion de l'Organe de règlement des différends réuni le 26 , I'OMC a autorisé l'UE à surtaxer de près de 4 milliards de dollars les importations américaines. Cette décision sanctionne les milliards de dollars de subventions sous forme de crédits d'impôts accordées à Boeing aux ÉtatsUnis d'Amérique.

Les États-Unis s'opposent à la Nigériane Okonjo-Iweala pour diriger l'OMC

Le 28 octobre 2020, a été présentée la candidature de Ngozi Okonjo-Iweala est soutenue par les présidents des trois principaux organes de I'OMC (Conseil général, Organe de règlement des différends et Organe d'examen des politiques commerciale). Washington s'y est opposé et préfère la Coréenne Yoo Myung-hee. La réu- nion du 9 novembre qui devait départager les candidates a été reportée en raison de ce conflit.

\section{Pas de consensus à l'OCDE}

Les 28 et 29 octobre 2020, les ministres des finances des États membres se sont réunis à I'OCDE (dont le secrétaire général doit être remplacé en 2021) pour discuter de la coordination de plans de relance pour l'économie après la pandémie de Covid-19. Tout ne fait pas consensus, comme la question de la fiscalité des GAFAM :les États-Unis ont suspendu leur participation;le premier piler sur la répartition des assiettes entre pays a fait l'objet d'un cadre général ;aucun seuil d'imposition minimum n'a été fixé pour le second pilier. La France souhaite une accélération des négociations alors que I'Allemagne est plutôt attentiste par rapport à l'évolution de la position américaine.

Aurélien BAUDU (fiscalité et procédure fiscale), Fabrice BIN (Europe, international), Jean-François CALMETTE (budget de l'État et des opérateurs),

Matthieu HOUSER (finances locales),

Michel LE CLAINCHE (comptabilité publique, management public, coordination), Yves TERRASSE (finances sociales).

Retrouvez sur www.gestionfinancespubliques. info :

- I'intégralité des REPÈRES d'actualité des finances et de la gestion publiques depuis 2015 - le 15 du mois, les REPÈRES d'actualité du mois précédent

- le 15 janvier 2021, le BEST OF des repères d'actualité de l'année 2020. 